\title{
Zur Stetigkeit der Lösung der adjungierten Gleichung bei Aufgaben der optimalen Steuerung mit Zustandsbeschränkungen
}

\section{Hoàng Xưîn Phứ}

Die Stetigkeit der Lösung der adjungierten Gleichung bei Aufgaben der optimalen Steuerung • mit Zustandsbeschränkungen wurde schon von anderen Autoren unter gewissen Bedingungen gezeigt, die oft nicht erfüllt sind. Hier wird die Stetigkeit dieser Lösung unter anderen Bedingungen-gezeigt. Weiterhin werden andere interessante Ergebnisse und Anwendungen erreicht.

Непрерывность ренения сопряженного уравнения в задачах оптимального управлени́я с ограничениями состояния уже доказана другими авторами, однако при условиях, часто не выполняющихся. В этой работе пепрерывность әтого решения доказывается при-других условия. Кроме этого работа содержит другие интересные результаты й применения.

The continuity of the solution of the adjoint equation in optimal control problems with state restrictions has already been shown by other authors under certain conditions that are often not fulfilled. Here the continuity of this solution is shown under different conditions. Furthermore, some other interesting results and applications are given.

\section{Problemstellang}

In dieser Arbeit betrachten wir die nachstehende.Aufgabe der:optinualen Steuerung mit festem Integrationsintervall:

$$
\begin{aligned}
& \mathcal{F}(x, u):=\int_{t_{0}}^{t_{1}} f(t, x(t), u(t)) d t \rightarrow \text { inf } ! \\
& \dot{x}(t)=\varphi(t, x(t), u(t)), \quad u(t) \in U \subset \mathbf{R}^{r}, \\
& g_{i}(t, x(t)) \leqq 0 \quad\left(t \in\left[t_{0}, t_{1}\right] ; i=1,2, \ldots, k\right), \\
& h_{0}\left(x\left(t_{0}\right)\right)=0, \quad h_{1}\left(x\left(t_{1}\right)\right)==^{\prime} .
\end{aligned}
$$

Dabei wird vorausgesetzt, daß die Funktionen und Abbildungen

$$
\begin{array}{ll}
f: \mathbf{R} \times \mathbf{R}^{n} \times \mathbf{R}^{r} \rightarrow \mathbf{R}, & g_{i}: \mathbf{R} \times \mathbf{R}^{n} \rightarrow \mathbf{R}, \\
\varphi: \mathbf{R} \times \mathbf{R}^{n} \times \mathbf{R}^{r} \rightarrow \mathbf{R}^{n}, & h_{l}: \mathbf{R}^{n} \rightarrow \mathbf{R}^{s_{1}} \quad(l=0,1)
\end{array}
$$

stetig und $f(t, \cdot, v), \varphi(t, \cdot, v), g_{i}$ und $h_{l}$ stetig differenzierb̄ar sind. Außerdem sei $U$ kompakt.

Als zulässige Steuerungen bezeichnen wir beliebige in $\left[t_{0}, t_{1}\right]$ rechtseitig stückweise stetige und in $t_{1}$ stetige Vektorfunktionen $u$, die Werte aus der Menge $U$ annehmen! Ein Paar $(x, u)$ heißt ein Proze $\beta$, falls $u$ eine zulässige Steuerung ist und alle Be-. dingungen in (1) erfüllt sind. Dabei heißt $x$ eine Zustandsfunktion und ihr Graph 
Trajektorie. $\left(x^{*}, \dot{u}^{*}\right)$ hèßt optimaler Prozeß, wenn für alle Prozesse $(\dot{x}, u)$ gilt $\mathcal{F}(x, u)$ $\geqq \mathcal{F}\left(x^{*}, u^{*}\right)$.

Für die Aufgabe (1) wurde von A. D. Toffe und V. M. ThcHomrov in [2] das folgende Pontrjaginsche Maximumprinzip bewiesen.

Satz 1: Sei $\left(x^{*}, u^{*}\right)$ ein optimaler Prozeß der Aufgabe (1). Dann existiert eine Zahl $\lambda_{0} \geqq 0$, Vektoren $l_{0} \in \mathbf{R}^{s_{0}}, l_{1} \in \mathbf{R}^{s_{1}}$, eine Vektorfunktion $p:\left[t_{0}, t_{1}\right] \rightarrow \mathbf{R}^{n}$ und $k$ aut den Mengen $T_{i}:=\left\{t \in\left[t_{0}, t_{1}\right] \mid g_{i}\left(t, x^{*}(t)\right)=0\right\}$ konzentrierte nichtnegative reguläre Maße $\mu_{i}(i=1,2, \ldots, k)$ derart, daß diese Größen nicht gleichzeitig verschwinden und folgendes gilt:

a) $p$ ist die Lösung der adjungiètten Gleichung

$$
\begin{aligned}
p^{\mathrm{T}}(t)= & h_{0}{ }^{\prime}\left(x^{*}\left(t_{0}\right)\right) \cdot l_{0}^{\mathrm{T}}+\int_{t_{0}}^{t} H_{\xi}\left(\tau, x^{*}(\tau), u^{*}(\tau), p(\tau), \lambda_{0}\right) d \tau \\
& +\sum_{i=1}^{k}-\int_{\left[\delta_{0}, t\right\}} g_{i \xi}\left(\tau, x^{*}(\tau)\right) d \mu_{i}, \\
p^{\mathrm{T}}\left(t_{1}\right)= & -h_{1}^{\prime}\left(x^{*}\left(t_{1}\right)\right) \cdot l_{1}^{\mathrm{T}} .
\end{aligned}
$$

b) Für fast alle $t$ aus $\left[t_{0}, t_{1}\right]$ ist die Gleichung

$$
\ddot{H}\left(t, x^{*}(t), u^{*}(t), p(t), \lambda_{0}\right)=\mathscr{H}\left(t, x^{*}(t), p(t), \hat{\lambda}_{0}\right)
$$

erfüllt.

Dabei ist $H(t, \xi, v, q, \lambda):=\varphi(t, \xi, v) \cdot q^{\mathrm{T}}-\lambda f(t, \xi, v) \quad$ und $\quad \mathscr{H}(t, \xi, q, \lambda):=$ $\sup H(\ell, \xi, v, q, \lambda)$.

Benutzt man das Pontrjaginsche Maximumprinzip, um zu untersuchen, ob ein vorgegebener Prozeß optimal sein kann, so treten nicht selten Schwierigkeiten auf, da die Maße $\mu_{i}$ und die Funktion $p$ izunächst unbekannt sind. Noch größer ist der Aufwand, weun ein (noch unbekannter) optimaler Prozeß $\left(x^{*}, u^{*}\right)$ erst zu bestimmen ist, weil dann sowohl $x^{*}, u^{*}$ als auch $p$ und $\mu_{i}$ unbekannt sind. Dabei.ist es schon eine große Erleichterung zu wissen, ob $p$ stetig ist, d. h., ob das Maß in einzelnen Punkten gleich Null-ist.

Was wissen wir bis jetzt iiber die Stetigkeit von $p$ ? Da jedes Maß $\mu_{i}$ auf der Randfläche

$$
\partial \dot{G}_{i}:=\left\{(t, \xi) \in\left[t_{0}, t_{1}\right] \times \mathbf{R}^{n} \mid g_{i}(t, \xi)=0\right\}
$$

konzentriert ist, ist die Funktion $p$ in $t$ stetig, wenn der Punkt $\left(t, x^{*}(t)\right)$ im Inneren des Zustandsbereiches

$$
G:=\left\{(t, \xi) \in\left[t_{0}, t_{1}\right] \times \mathbf{R}^{n} \mid g_{i}(t, \xi) \leqq 0(i=1,2, \ldots, k)\right\}
$$

liegt. Die Unstetigkeit von $p$ kann also nur auf dem Rande des Zustandsbereichs $G$, auftreten. Außerdem ist $p$ wegen der Regularität der Maße $\mu_{i}$ in $\left[t_{0}, t_{1}\right]$ linksseitig stetig (vgl. [2]).

W. G. BoltJarskI zeigte in [1] die Stetigkeit von $p$ unter einer Zusatzbedingung, die er „Bedingung der allgemeinen Lage ${ }^{6 /}$ nennt. Wenn ।

$$
\begin{aligned}
& \frac{\partial}{\partial t} g_{i}(t, \xi)=0 \quad \text { für alle } \quad(t, \xi) \in G \quad(i=1,2, \ldots, k), \text { und } \\
& U:=\left\{v \in \mathbf{R}^{r} \mid g_{j}(v) \leqq 0 \quad(j=k+1, k+2, \ldots, m)\right\}
\end{aligned}
$$


gilt, d. h. $U$ durch $m-k$ vorgegebene Funktionen $g_{k+1}, g_{k+2}, \ldots, g_{m}$ definiert wird, dann besagt diese Bedingung, daß die Vektoren

$$
\operatorname{grad}_{v} g_{i}(t, \xi) \text { und } \operatorname{grad}_{v} g_{j}(v) \text {, }
$$

im . Falle $g_{i}^{\prime}(l, \xi)=0$ und $g_{j}(v)=0$ linear unabhängig sein sollen. Weil nun aber $\operatorname{grad}_{v} g_{i}(t, \xi)=(0,0, \ldots, 0)$ für $i=1,2, \ldots, k$ und $(t, \xi) \in G$ gilt, ist diese Bedingung verletzt.

H. W. KroвLOCH zeigte in [3] die Stetigkeit von $p$ für solche Aufgaben mit einer Zustandsbeschränkung, d. h. $k=1$, allerdings unter den Bedingungen, da $ß$

$$
\begin{aligned}
& \frac{d}{d t} g_{1}\left(z_{1}, x\left(z_{1}\right)\right)>0 \quad \text { für jede Aufsprungstelle } z_{1} \text { und } \\
& \frac{d}{d t} g_{1}\left(z_{2}, x\left(z_{2}\right)\right)<0 \quad \text { für jede Absprungstelle } z_{2}
\end{aligned}
$$

gilt und daß jede Aufsprungstelle zugleich eine Absprungstelle ist. Diese Forderungen sind bei unseren im Abschnitt 3 genannten Aufgaben (13) und (14) nicht erfüllt.

In dieser Arbeit wird die Stetigkeit der Funktion $p$ unter anderen Bedingüngen gezeigt, und anschließend werden einige Anwendungen dieser Untersuchungen betrachtet.

Definition: Wenn für ein $i, 1 \leqq i \leqq \hat{k}$, und $z_{1}, z_{2}$ aus $\left[t_{0}, t_{1}\right]$ gilt

$$
\begin{aligned}
& z_{1} \leqq z_{2}, \quad g_{i}(t, x(t))=0 \text { für alle } t \in\left[z_{1}, z_{2}\right] \text {. und } \\
& \text { für beliebiges } \varepsilon>0 \text { existiert ein } t \in\left(z_{1}-\varepsilon, z_{1}\right) \text { nit } \\
& g_{i}(t, x(t))<0 \text {, und ein } t \in\left(z_{2}, z_{2}+\varepsilon\right) \text { mit } g_{i}(t, x(t))<0 \text {, }
\end{aligned}
$$

dann heißt $z_{1}$ eine $g_{i}$-Aufsprungstelle, $z_{2}$ eine $g_{i}$-Absprungstelle und jedes $t \in\left(z_{1}, z_{2}\right)$ eine $g_{i}$-Verweilstelle von $x$.

Diese Begriffe entlehnen wir H. W. Кмовцосн [3], sie werden aber bei uns in einem umfassenderen Sinne gebraucht: H. W. KNoвLOCH forderte, daß es nür endlich viele Aufsprung- und Absprungstellen gibt, was bei uns nicht der Fall zu sein braucht.

\section{Einige Hilfsmittel}

In diesem Abschnitt stellen wir einige Lemmata zusammen, die im nächsten Abschnitt angewandt werden.

Lemma 1: Gegeben seien zwei Funktionen $b: Z \times U \rightarrow \mathbf{R}^{1}$ und $a: Z \rightarrow \mathbf{R}^{1}$, wolvei $Z \subset \mathbf{R}^{1}, U \subset \mathbf{K}^{r}$ kompakt und $b(t, \cdot)$ stetig für alle $t \in Z$ sind. Ferner sei

$$
\begin{aligned}
& F_{1}(t):=\{v \in U \mid b(t, v)=a(t)\}, \\
& F_{2}(t):=\{v \in U \mid b(t, v) \leqq a(t)\} \quad \text { und } \quad F_{3}(t):=\{v \in U \mid b(t, v) \geqq a(t)\} .
\end{aligned}
$$

Sind dann $b\left(\cdot, v_{1}\right)$ und $a$ in $z \in \operatorname{int} \dot{Z}$ linksseitig (bzw. rechlsseitig) stetig, so sind alle Abbildungen $F_{j}(j=1,2,3)$ in $z$ linksseitig (bzw. rechtsseitig) oberhalbstetig. 
Beweisidee: Es seien $b(\cdot, v)$ und $a$ in $z \in$ int $Z$ linksseitig stetig. Angenommen, $F_{1}$ wäre in $z$ nicht linksseitig oberhalbstetig. Dann existiert ein $\varepsilon>0$, so da 13 sich für alle $\delta>0$ ein $t \in Z \cap(z-\delta, z)$ und $v(t) \in F_{1}(t)$ mit $v(t) \notin F_{1}(z)+V(\varepsilon)$ finden läßt, wobei $V(\varepsilon)$ eine $\varepsilon$-Nullumgebung in $\mathbf{R}^{r}$ ist. Man kann eine Folge $\left\{z_{i}\right\}$ konstruieren mit

$$
\begin{aligned}
& z: \in(z-\delta, z) \text { für alle } i, \lim _{i \rightarrow \infty} z_{i}=z, \\
& v\left(z_{i}\right) \in F_{1}\left(z_{i}\right), \quad v\left(z_{i}\right) \notin F_{1}(z)+V(z) .
\end{aligned}
$$

Weiterhin wird gezeigt, daß

$$
\left|b\left(z, v\left(z_{i}\right)\right)-a(z)\right| \geqq \gamma \quad(i=1,2, \ldots)
$$

für ein $\gamma>0$ gilt (vgl. [6]). Wegen der Kompaktheit von $U$ existiert eine Teilfolge $\left\{z_{i^{\prime}}\right\}$, mit $\lim _{i^{\prime} \rightarrow \infty} v\left(z_{i^{\prime}}\right)=\bar{v} \in U$. Für diese Teilfolge gilt wegen der linksseitigen, Stetigkeit von $b(\cdot, v)$ und $a$ und wegen der Stetigkeit von $b(t, \cdot)$

$$
\begin{aligned}
0 & =\lim _{i^{\prime} \rightarrow \infty}\left(\left|b\left(z, v\left(z_{i^{\prime}}\right)\right)-b\left(z_{i^{\prime}}, v\left(z_{i^{\prime}}\right)\right)\right|+\left|a\left(z_{i^{\prime}}\right)-a(z)\right|\right) \\
& \geqq \lim _{i^{\prime} \rightarrow \infty}\left|b\left(z, v\left(z_{i^{\prime}}\right)\right)-b\left(z_{i^{\prime}}, v\left(z_{i^{\prime}}\right)\right)+a\left(z_{i^{\prime}}\right)-a(z)\right| \\
& =\lim _{i^{\prime} \rightarrow \infty}\left|b\left(z, v\left(z_{i^{\prime}}\right)\right)-a(z)\right| \geqq \gamma,
\end{aligned}
$$

im Widerspruch zu $0<\gamma$. Also ist die Annahme falsch, und deshalb muß $F_{1}$ in diesem Fall linksseitig oberhalbstetig sein. Für $F_{2}$ und $F_{3}$ verläuft der Beweis ana$\log$

Dieses Lemma wird später bei der Auswertung der Gleichung (3) im Pontrjaginschen Maximumprinzip cine wichtige Rolle spielen. Es gestattet die Eigenschaften optimaler Prozesse auch an solchen Stellen zu ergründen, in denen (3) nicht gilt:

Es seien

$$
\begin{aligned}
& p_{z}(t):= \begin{cases}p(t) & \text { für } t \neq z, \\
p(z+0) & \text { für } t=z\end{cases} \\
& \tilde{H}(t, v):=H\left(t, x^{*}(t), v, p(t), \lambda_{0}\right), \quad \tilde{H}_{z}(t, v):=H\left(t, x^{*}(t), v, p_{z}(t), \lambda_{0}\right), \\
& \tilde{H}(t):=\mathscr{H}\left(t, x^{*}(t), p(t), \lambda_{0}\right), \quad \tilde{\mathscr{H}}_{z}(t):=\mathscr{H}\left(t, x^{*}(t), p_{z}(t), \lambda_{0}\right) .
\end{aligned}
$$

Dann gilt die folgende Aussage.

Lemma 2: $\tilde{H}(\cdot, v)$ und $\tilde{\mathscr{H}}$ sind in $t=z$ linksseitig stetig, $\tilde{H}_{z}(\cdot, v)$ und $\tilde{\mathscr{H}}_{z}$ sind in $t=\dot{z}$ rechtsseitig stetig.

Der Beweis für dieses Lemma ist ganz ähnlich dem von B. N. Pš́nxı̌xyJ [8: Kap: 3] für den stetigen Fall zu führen (s. [6]).

Definition: Es sei $y(t):=(t, x(t)) \in G$. Dann heißt $d_{i}$ mit

$$
d_{i}(t):=\operatorname{Min}\left\{\|\dot{y}(t)-\eta\| \dot{\mid \eta} \in \partial G_{i}\right\}
$$

Abstandsfunktion zwischen der Trajektorie $y$ und der Randfläche $\dot{\partial} G_{i}$ und jeder Punkt $y_{i}(t)$ nit

$$
y_{i}(t) \in \partial G_{i} \text { und }\left\|\dot{y}_{i}(t)-y(t)\right\|=d_{i}(t)
$$

' heißt nächster, $F u \beta p u n k t$ von $y(t)$ auf $\partial G_{i}$. . 
Lem ma $3: d_{i}(i=1,2, \ldots, k)$ ist stetig und fast überall differenzierbar.

Beweis: Aus der-Definition folgt für $z_{1}, z_{2} \in\left[t_{0}, t_{1}\right]$

$$
\begin{aligned}
\left|d_{i}\left(z_{1}\right)-d_{i}\left(z_{2}\right)\right| & \leqq\left\|y\left(z_{1}\right)-y_{i}\left(z_{2}\right)\right\|-\left\|y\left(z_{2}\right)-y_{i}\left(z_{2}\right)\right\| \\
& \leqq\left\|y\left(z_{1}\right)-y\left(z_{2}\right)\right\| \leqq\left|z_{1}-z_{2}\right|+\left\|x\left(z_{1}\right)-x\left(z_{2}\right)\right\| .
\end{aligned}
$$

Wegen der Kompaktheit von $U$ und der Stetigkeit von $x$ und $\varphi$ existiert ein $K$ mit $\|\varphi(t, x(t), \ddot{u}(t))\| \leqq K$ für alle $t \in\left[t_{0}, t_{1}\right]$. Daraus folgt

$$
\left\|x\left(z_{1}\right)-x\left(z_{2}\right)\right\| \leqq \int_{z_{1}}^{z_{1}}\|\varphi(t, x(t), u(t))\| d t \leqq K \cdot\left|z_{2}-z_{1}\right| .
$$

Damit gilt $\left|d_{i}\left(z_{1}\right)-d_{i}\left(z_{2}\right)\right| \leqq(K+1) \cdot\left|z_{2}-z_{1}\right|, \quad$ d. h., $d_{i}$ ist Lipschitz-stetig in $\left[t_{0}, t_{1}\right]$. Deshalb ist $d_{i}$ in $\left[t_{0}, t_{1}\right]$ stetig und fast überall differenzierbar (vgl. H. RADEMACHER [9])

Lémma 4: Sèi $z \in\left[t_{0}, t_{1}\right]$ mit $g_{i}(z \vec{x}(z))=0$. Für alle $\omega>0$ giltt:

a) $\dot{d}_{i}(t)^{\prime} \leqq 0$ auf einer Menge von positivem $M a \beta$ im Intervall $(z-\omega, z)$,

b) $\dot{d}_{i}(t) \geqq 0$ auf einer Menge von positivem $M a \beta$ im Intervall $(z, z+\omega)$.

Beweis: Wäre $\dot{d}_{i}(t)>0$ fast überall in $(z-\omega, z)$, dann gälte für ein $z_{1} \in(z-\omega, z)$ mit. $d_{i}\left(z_{1}\right)>0$ die Ungleichung

$$
d_{i}(z)>d_{i}(z)-d_{i}\left(z_{1}\right)=\int_{z_{1}}^{z} \dot{d}_{i}(t) d t>0,
$$

im Widerspruch zu $d_{i}(z)=0$. Also muB a) wahr sein. Der Beweis für b) verläuft analog

Bei unserer Betrachtung fordern wir sogar:

Für alle $\omega>0$ existieren zwei Mengen $\Omega_{1} \subset(z-\omega, z)$ und $\Omega_{2} \rightleftarrows(z, z+\omega)$ - von positivem Maß mit der Eigenschaft:

$\dot{d}_{i}(t) \leqq 0$ für alle $t \in \Omega_{1}$ und $i \in I(z, x)$ und

$\dot{d}_{i}(t) \geqq 0$ fiir alle $t \in \dot{\Omega}_{2}$ und $i \in I(z, x)$.

Dabei ist $I(z, x)$ die Menge der aktiven Indizes in $z$ bez. der Zustandsfunktion $x$ und wie folgt definiert:

$$
I(\dot{z} ; x):=\left\{i \in\{1,2, \ldots, k\} \mid g_{i}(z, x(z))=0\right\} .
$$

Die Forderung (4) ist in vielen Fällen erfïllt. Zum Beispiel folgt aus Lenıma 4, daß (4) gilt, wenn es höchstens ein $i_{1}$ und ein $i_{2}$ aus $I(z ; x)$ gibt, für die $z$ eine $g_{i_{1}}$-Auf-

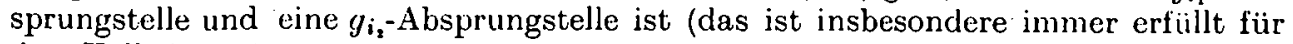
den Fall $k=1$ ).

Nun untersuchen wir’die nächsten Fußpunkte. Für ein beliebigès $t \in\left[t_{0}, t_{1}\right]$ kann es mehrere solcher Punkte geben'. Wir betrachten deshalb die folgende lokale Lip- 
schitz-Bedingung:

Für alle $\eta \in \partial G$ und $i \in I(\eta)$ existieren $\varepsilon_{i}(\eta)>0$ und $M_{i}(\eta)>0$, so da $B$ für $\eta^{\prime}, \eta^{\prime \prime} \in U_{e_{i}}(\eta) \cap \partial G_{i}$ gilt

$$
\left\|\mathfrak{R}_{i}\left(\eta^{\prime}\right)-\mathfrak{R}_{i}\left(\eta^{\prime \prime}\right)\right\| \leqq M_{i}(\eta) \cdot\left\|\eta^{\prime}-\eta^{\prime \prime}\right\|,
$$

wobei $U_{\varepsilon_{i}}(\eta)$ eine $\varepsilon_{i}(\eta)$-Umgebung vọn $\eta$ in $\mathbf{R}^{n+1}$ und $\mathfrak{R}_{i}$ der äußere Normalenvektor der Randfläche $\partial G_{i}$ ist.

Mit ihrer Hilfe gelingt die folgende Aussage.

Lemma 5: Für alle $z \in\left[t_{0}, t_{1}\right]$ mit $\dot{g}_{i}(y(z)) \stackrel{\perp}{=}$ existiert eine Umgebung von $z$, in der die Abbildung $t . y_{i}(t)$ eindeutig, stetig, und fast überall differenzierbar ist.

Beweis: Wir setzen

$$
\varepsilon:=\operatorname{Min}\left\{\varepsilon_{i}(y(z)), \frac{1}{M_{i}(y(z))}\right\}>0 \text {. }
$$

Wegen der Stetigkeit von $d_{i}$ und $y$ sówie wegen. $d_{i}(z)=0$ existiert zu diesem $\varepsilon$ solch ein $\delta=\delta(\varepsilon)>0$, daß $|t-z|<\delta$ zu $\|y(t)-y(z)\|<\frac{\varepsilon}{2}$ und $d_{i}(t)<\frac{\varepsilon}{2}$ führt. Sei , nun $y_{i}(t)$ ein beliebiger nächster Fußpunkt von- $y(t)$ auf der'Randfläche $\partial G_{i}$. Dann gilt nach der Definition von $d_{i}$ und mit Hilfe der Dreiecksungleichung

$$
\left\|y_{i}(t)-y(z)\right\| \leqq d_{i}(t)+\|y(t)-y(z)\|<\varepsilon \leqq \varepsilon_{i}(y(z)),
$$

d. h.' $y_{i}(t) \in U_{\varepsilon_{i}}(y(z)) \cap \partial G_{i}$ für $|t-z|<\delta$. Deshalb folgt aus (5)

$$
\left\|\mathfrak{R}_{i}\left(y_{i}\left(z_{1}\right)\right)-\mathfrak{R}_{i}\left(y_{i}\left(z_{2}\right)\right)\right\| \leqq M_{i}(y(z)) \cdot\left\|y_{i}\left(z_{1}\right)-y_{i}\left(z_{2}\right)\right\|
$$

für $\left|z_{j}-z\right|<\delta, j=-1,2$. Wegen der Definition von $d_{i}$ und

$$
y_{i}\left(z_{j}\right)=y\left(z_{j}\right)+d_{i}\left(z_{j}\right) \cdot \mathfrak{R}_{i}\left(y_{i}\left(z_{j}\right)\right) \quad(j=1,2)
$$

gilt weiterhin

$$
\begin{aligned}
& \left\|y_{i}\left(z_{1}\right)-y_{i}\left(z_{2}\right)\right\| \\
& \leqq\left\|y\left(z_{1}\right)-y\left(z_{2}\right)\right\|+d_{i}\left(z_{1}\right) \cdot\left\|\Re_{i}\left(y_{i}\left(z_{1}\right)\right)-\Re_{i}\left(y_{i}\left(z_{2}\right)\right)\right\|+\left|d_{i}\left(z_{1}\right)-d_{i}\left(z_{2}\right)\right| \\
& \leqq 2\left\|y\left(z_{1}\right)-y\left(z_{2}\right)\right\|+d_{i}\left(z_{1}\right) M_{i}(y(z))\left\|y_{i}\left(z_{1}\right)-y_{i}\left(z_{2}\right)\right\|
\end{aligned}
$$

für: $\left|z_{j}-z\right|<\delta, j=1,2$. Weil

$$
d_{i}\left(z_{1}\right) M_{i}(y(z))<\frac{\varepsilon}{2} \cdot M_{i}(y(z)) \leqq \frac{1}{2 M_{i}(y(z))} M_{i}(y(z))=\frac{1}{2}
$$

ist, ergibt sich daraus $\left\|y_{i}\left(z_{1}\right)-y_{i}\left(z_{2}\right)\right\| \leqq 4\left\|y\left(z_{1}\right)-y\left(z_{2}\right)\right\|$ für $\left|z_{j}-z\right|<\delta, j=1,2$. Da $y_{i}\left(z_{j}\right)$ ein beliebiger nächster Fußpunkt von $y\left(z_{j}\right)$ ist, folgt die Eindeutigkeit von $y_{i}(t)$ für $|t-z|<\delta$ aus der letzten Ungleichung (für $z_{1}=z_{2}$ ).

Genau. wie im Beweis zu Lemma 3 existiert ein $\bar{K}>0$ mit $\left\|y\left(z_{1}\right)-y\left(z_{2}\right)\right\|$ $\leqq \bar{K} \cdot\left|z_{1}-z_{2}\right|$. Daraus folgt ||$y_{i}\left(z_{1}\right)-y_{i}\left(z_{2}\right) \| \leqq 4 \bar{K}\left|z_{1}-z_{2}\right|$ für $\left|z_{j}-z\right|<\delta(j=1$, $2)$, d. h., $y_{i}$ ist in der $\delta$-Umgebung von $z$ Lipschitz-stetig. Deshalb ist $y_{i}$ dort fast überall differenzierbar (vgl. bei H. RADEMACHER [9])

Aus Lemma 3, Lemima 5 und der Definition von $d_{i}$ folgt

$$
\dot{d}_{i}(t)=-\frac{1}{\left\|\operatorname{grad} g_{i}\left(y_{i}(t)\right)\right\|}\left(\dot{y}(t)-\dot{y}_{i}(t)\right) \operatorname{grad} g_{i}\left(y_{i}(t)\right)
$$


Wegen

$$
\begin{aligned}
& \dot{y}(t) \operatorname{grad} g_{i}\left(y_{i}(t)\right)=0, \\
& \dot{y}(t)=(1, \dot{x}(t))=(1, \varphi(t, x(t), u(t))), \\
& \operatorname{grad} g_{i}\left(y_{i}(t)\right)=\left(\begin{array}{l}
g_{i t}\left(y_{i}(t)\right) \\
g_{i \xi}\left(y_{i}(t)\right)
\end{array}\right)
\end{aligned}
$$

folgt aùs (4) für alle $\omega>0$ die Existenz zweier Mengen $\Omega_{1} \subset(z-\omega, z)$ und $\Omega_{2}$ $\subset(z, z+\omega)$ von positivem Maß mit der Eigenschaft:

$$
\begin{array}{lllll}
\varphi(t, x(t), u(t)) g_{i \xi}\left(y_{i}(t)\right) \geqq-g_{i t}\left(y_{i}(t)\right) & \text { für } t \in \Omega_{1} & \text { und } & i \in I(z, x), \\
\varphi(t, x(t), u(t)) g_{i \xi}\left(y_{i}(t)\right) \leqq-g_{i t}\left(y_{i}(t)\right) & \text { für } & t \in \Omega_{2} & \text { und } & i \in I(z, x) .
\end{array}
$$

Wenn wir für den optimalen Prozeß $\left(x^{*}, u^{*}\right)$ dịe Méngen ,

und

$$
W_{1}(t):=\left\{v \in U \mid \varphi\left(t, x^{*}(t), v\right) g_{i \xi}\left(y_{i}^{*}(t)\right) \geqq-g_{i \iota}\left(y_{i}^{*}(t)\right) \forall i \in I\left(z, x^{*}\right)\right\}
$$

$$
W_{\cdot 2}(t):=\left\{\dot{v} \in U \mid \varphi\left(t, x^{*}(t), v\right) g_{i \xi}\left(y_{i}^{*}(t)\right) \leqq-g_{i t}\left(y_{i}^{*}(i)\right) \forall i \in I\left(z, x^{*}\right)\right\}
$$

definieren, wobei $y_{i}{ }^{*}(t)$ ein nächster Fußpunkt von $y^{*}(t)=\left(t, x^{*}(t)\right)$ auf $\partial G_{i}$ ist, dann lautet die letzte Eigenschaft folgendermaßen.

Lemma 6: Wenn $\left(x^{*}, u^{*}\right)$ der Bedingung (4) genügt, dann ist $u^{*}(t) \in W_{1}(t)$ für $t \in \Omega_{1}$ und $u^{*}(t) \in W_{2}(t)$ für $t \in \Omega_{2}$ (mit den in (4) definierten $\left.\Omega_{1}, \Omega_{2}\right)$.

\section{Zur Stetigkeit von $p$ und andere Hauptergebnisse}

Zunächst führen wir einige Definitionen ein:

$$
\begin{aligned}
& \mathscr{H}^{-v}(t):=\left\{v \in U \mid \tilde{H}(t, v)=\tilde{\mathscr{H}}_{1}(\bar{t})\right\}, \quad \mathscr{H}_{z}^{-v}(t):=\left\{v \in \dot{U} \mid \tilde{H}_{z}(t, v)=\tilde{\mathscr{H}}_{z}(t)\right\}, \\
& M_{1}(t):=W_{1}(t) \cap \mathscr{H}^{-v}(t), \quad M_{2}(t):=W_{2}(t) \cap \mathscr{H}_{z}^{-v}(t) .
\end{aligned}
$$

Alle im Abschnitt 2. geführten Betrachtungen sind eine Vorbereitung für Lémma 7.

Lemma 7 : Die Bedingungen (4) und (5) seien erfüllt und es sei $z \in\left(t_{0}, t_{1}\right)$ mit. $I\left(z, x^{*}\right) \neq \emptyset$. Dann gilt $M_{1}(z) \neq \emptyset$ und $M_{2}(z) \neq \emptyset$.

Beweis: a) Nach Lemma 2 sind $\tilde{H}(\cdot, v)$ und $\tilde{\mathscr{H}}$ in $t=z$ linkssèitig stetig und $\tilde{H}_{z}$ und $\tilde{\mathscr{H}}_{z}$ in $t=z$ rechtsseitig stetig. Deshalb gilt nach Lemma 1 , daß $\mathscr{H}^{-v}$ in $t=z$ linksseitig oberhalbstetig und $\mathscr{H}_{z}^{-v}$ in $t=z$ rechtsseitig oberhalbstetig sind. Nach Lemma 5 ist $y_{i}^{*}$ in einer hinreichend kleinen Umgebung von $z$ stetig. Wegen der Stetigkeit von $x^{*}$ und $\varphi$ und der stetigen Differenzierbarkeit von $g_{i}$ sind die Funktionen

$$
t \mapsto\left(t, x^{*}(t), v\right) g_{i \xi}\left(y_{i}^{*}(t)\right) \quad \text { und } \quad t \mapsto-g_{i s}\left(y_{i}^{*}(t)\right)
$$

für alle $i$ ebenso stetig in dieser Umgebung. Deshalb folgt aus Lemma 1, daB.

$$
W_{1 i}(t):=\left\{v \in U \mid \varphi\left(t, x^{*}(t), v\right) g_{i \xi}\left(y_{i}^{*}(t)\right) \geqq-g_{i t}\left(y_{i}^{*}(t)\right)\right\}
$$

für $i \in I\left(z, x^{*}\right)$ in $t^{\prime}=z$ oberhalbstetig ist. Damit wird $W_{1}(t)$ folgendermaßen gebildet

$$
W_{1}(t)=\bigcap_{i \in I\left(z, x^{*}\right)} W_{1 i}(t)
$$


wobei nur endlich viele Mengen $W_{1 i}(t)$ zum Durchschnitt gebracht werden. Folglich ist $W_{1}$ in $t=z$ ebenfalls oberhalbstetig. Analog folgt die Oberhalbstetigkeit von $W_{2}$ in $t=z$. Aus der Definition folgt nun die linksseitige Oberhalbstetigkeit. von $M_{1}$ und die rechtsseitige Oberhalbstetigkeit von $M_{2}$ in $t=z$.

b) Die Gleichung (3) bedeutet nach der Definition von $\mathscr{H}^{-v}$

$$
u^{*}(t) \in \mathscr{H}^{-v}(t) \text { fast überall in }\left[t_{0}, t_{1}\right] .
$$

Nach Lèmma 6 existiert zu jedem $\omega>0$ eine Menge $\Omega_{1}^{\prime}$ mit

$$
u^{*}(t) \in W_{1}(t) \cap \mathscr{H}^{-v}(t)=M_{1}(t) \text { für alle } t \in \Omega_{1}^{\prime},
$$

wobei $\Omega_{1}^{\prime}$ gleich dem Durchschnitt von $\Omega_{1}$ mit der Menge aller $t$ ist, für die $u^{*}(t)$ $\epsilon \mathscr{H}^{-v}(t)$ gilt. Weil $u^{*}(t) \in \mathscr{H}^{-v}(t)$ fast überall in $\left[t_{0}, t_{1}\right]$ gilt, ist das Maß von. $\Omega_{1}^{\prime}$ gleich dem Ma $\beta$ von $\Omega_{1}$, also größer als Null. Das bedeutet, daß zu jedem $\omega>0$ eine Menge $\Omega_{1}{ }^{\prime} \subset(z-\omega, z) \cap\left[t_{0}, t_{1}\right]$ von positivem Maß mit $M_{1}(t) \neq \emptyset$ für $t \in \Omega_{1}{ }^{\prime}$ existiert. Weil sich $p$ und $p_{z}$ nur in $t=z$ unterscheiden, gilt auch $u^{*}(t) \in \mathscr{H}_{z}^{-v}(t)$ fast iiberall in $\left[t_{0}, t_{1}\right]$. Dann kann analog gezeigt werden, da $\beta$ zu jedem $(\omega>0$ eine Menge $\Omega_{2}^{\prime} \subset(z, z+\omega) \cap\left[t_{0}, t_{1}\right]$ von positiven Maß mit $M_{2}(t) \neq \emptyset$ für $t \in \Omega_{2}^{\prime}$ existiert:

c) In a) wurde gezeigt, da $M_{1}$ in $t=z$ linksseitig oberhalbstetig ist, d. h. für alle $\varepsilon>0$ existiert ein $\omega>0$, so daß $M_{1}(t) \subset M_{1}(z)+V(\varepsilon)$ für $t \in(z-\omega, z)$ gilt, wobei $V(\varepsilon)$ eine Nullumgebung in $\mathbf{R}^{r}$ ist. Nach b) existiert ein $\Omega_{1}{ }^{\prime} \subset(\dot{z}-\omega, z)$ von positivem Maß mit $M_{1}(t) \neq \emptyset$ für $t \in \Omega_{1}^{\prime}$. Daraus folgt $M_{1}(z)+V(\varepsilon) \neq \emptyset$ für alle $\varepsilon>0$. Folglich ist $M_{1}(z) \neq \emptyset$. Anạlog läßBt.sich $M_{2}(z) \neq \emptyset$ zeigen

Lemma 7 ist das wichtigste Hilfsmittel zum Beweis des folgenden Satzes.'Damit die Ausdrücke übersichtlicher sind, wollen' wir in wciteren schreiben :

$$
\begin{aligned}
\tilde{H}(z+0): & =\tilde{H}_{z}(z)=\mathscr{H}\left(z, x^{*}(z), p(z+0), \lambda_{0}\right), \\
\mathscr{H}^{-v}(z+0): & =\mathscr{H}_{z}{ }^{-v}(z)=\left\{v \in U \mid H\left(z, x^{*}(z), v, p(z+0), \lambda_{0}\right)\right. \\
& \left.=\mathscr{H}\left(z, x^{*}(z), p(z+0), \lambda_{0}\right)\right\} .
\end{aligned}
$$

Satz'2: $\left(x^{*}, u^{*}\right)$ sei ein optimaler Proze $\beta$ der Aufgabe (1) mit (4) und (5), $z \in\left(t_{0}, t_{1}\right)$ mit $I\left(z, x^{*}\right) \neq 0$. Dann gilt:

$$
\begin{aligned}
& \text { a) } \tilde{\mathscr{H}}(z)=\tilde{\mathscr{H}}(z+0)+\sum_{i \in I\left(z, x^{*}\right)} \mu_{i}(\{z\}) g_{i t}\left(z, x^{*}(z)\right) \text {; } \\
& M_{1}(z) \subset \mathscr{H}^{-v}(z+0) \text { und } M_{2}(z) \subset \mathscr{H}^{-v}(z) .
\end{aligned}
$$

- Aus $v_{1} \in M_{1}(z)$ folgt für alle $i \in I\left(z, x^{*}\right)$

$$
\mu_{i}(\{z\})\left[g_{i t}\left(z, x^{*}(z)\right)+\varphi\left(z, x^{*}(z), v_{1}\right) g_{i \xi}\left(z, x^{*}(z)\right)\right]=0,
$$

áus $v_{2} \in M_{2}(z)$ folgt für alle $i \in I\left(z, x^{*}\right)$

$$
\mu_{i}(\{z\})\left[g_{i \ell}\left(z, x^{*}(z)\right)+\varphi\left(z, x^{*}(z), v_{2}\right) g_{i \xi}\left(z, x^{*}(z)\right)\right]=0 .
$$

b) Existiert ein $\bar{v} \in M_{1}(z)$ u $M_{2}(z)$ mit

$$
g_{i l}\left(z ; x^{*}(z)\right)+\varphi\left(z, x^{*}(z), \bar{v}\right) g_{i \xi}\left(z, x^{*}(z)\right) \neq 0,
$$

so ist $\mu_{i}(\{z\})=0$.

c) Es sei für alle $v \in \partial U$ und alle $i \in I\left(z, x^{*}(z)\right)$

$$
g_{i t}\left(z, x^{*}(z)\right)+\varphi\left(z, x^{*}(z), v\right) \cdot g_{i \xi}\left(z, \dot{x}^{*}(z)\right) \neq 0 .
$$


Außerdem existiere für alle $v \in$ int $U$ ein $j=j(v)(1 \leqq j \leqq r)$, so daß entweder

$$
\varphi_{v,}\left(z, x^{*}(z), v\right) g_{i \xi}\left(z, x^{*}(z)\right)>0 \text { für alle. } i \in I\left(z, x^{*}\right)
$$

oder

$$
\varphi_{u, j}^{\prime}\left(z, x^{*}(z), v\right) g_{i \xi}\left(z, x^{*}(z)\right)<0 \quad \text { für alle } i \in I\left(z, x^{*}\right)
$$

ist. 'Dann ist $p$ in $z$ stetig (d. h. $\mu_{i}(\{z\})=0$ für alle $i \in I\left(z, x^{*}\right)$.

d) Gilt (6) und (7) für alle $z \in\left(t_{0}, t_{1}\right)$, dann ist $p$ in $\left[t_{0}, t_{1}\right]$ stetig.

Bèweis: Wir beschränken uns darauf, a) und c) zu zeigen. b) und d) lassen sich einfach daraus folgern.

a) Nach Lemma 7 ist $M_{1}(z) \neq \emptyset$. 'Für cin beliebiges $v_{1} \in M_{1}(z)$ gilt danñ wegen

$$
p^{\mathrm{T}}(z+0)-p^{\mathrm{T}}(z)=\sum_{i \in I\left(z, x^{*}\right)} \mu_{i}(\{z\}) g_{i \xi}\left(z, x^{*}(z)\right)
$$

(vgl. (2)) die Beziehung

$$
\begin{aligned}
\tilde{\mathscr{H}}(z)= & \varphi\left(z, x^{*}(z), v_{1}\right) p^{\mathrm{T}}(z)-\lambda_{0} f\left(z, x^{*}(z), v_{1}\right) \\
\doteq & {\left[\varphi\left(z, x^{*}(z), v_{1}\right) p^{\mathrm{T}}(z+0)-\lambda_{0} f\left(z, x(z), v_{1}\right)\right] } \\
& \quad-\sum_{i \in I\left(z, x^{*}\right)} \mu_{i}(\{z\}) \varphi\left(z, x^{*}(z), v_{1}\right) g_{i \xi}\left(z, x^{*}(z)\right) \\
= & H\left(z, x^{*}(z), v_{1}, p(z+0), \lambda_{0}\right) \\
& -\sum_{i \in I\left(z, x^{*}\right)} \mu_{i}(\{z\}) \varphi\left(z, x^{*}(z), v_{1}\right) g_{i \xi}\left(z, x^{*}(z)\right)
\end{aligned}
$$

Einerseits ist

$$
\ddot{H}\left(z, x^{*}(z), v_{1}, p(z+0), \lambda_{0}\right) \leqq \tilde{\mathscr{H}}(z+0),
$$

andererseits folgt aus $v_{1} \in W_{1}(z)$

$$
\varphi\left(z, x^{*}(z), v_{1}\right) g_{i \xi}\left(z, x^{*}(z)\right) \geqq-g_{i l}\left(z, x^{*}(z)\right)
$$

für alle $i \in I\left(z^{\prime}, x^{*}\right)^{\prime}$. Weil $\mu_{\mathbf{i}}(\{z\}) \geqq 0$ für alle $i$ ist, gilt wegen der letzten Ungleichung

$$
\mu_{i}(\{z\}) \varphi\left(z, x^{*}(z), v_{1}\right) g_{i \xi}\left(z, x^{*}(z)\right) \geqq-\mu_{i}(\{z\}) g_{i t}\left(z, x^{*}(z)\right)
$$

fiir alle $i \in I\left(z, x^{*}\right)$ und damit

$$
\sum_{i \in I\left(z, x^{*}\right)} \mu_{i}(\{z\}) \varphi\left(z, x^{*}(z), v_{1}\right) g_{i \xi}\left(z, x^{*}(z)\right) \geqq \sum_{i \in I\left(z, x^{*}\right)}-\mu_{i}(\{z\}) g_{i t}\left(z, x^{*}(z)\right) .
$$

Also haben wir

$$
\text { - } \tilde{\mathscr{H}}(z) \leqq \tilde{H}(z+0)+\sum_{i \in I\left(z, x^{*}\right)} \mu_{i}(\{z\}) g_{i l}\left(z, x^{*}(z)\right) \text {. }
$$

Nach Lemma 7 ist auch $M_{2}(z) \neq 0$. Sei $v_{2} \in M_{2}(z)$ beliebig, dann gilt analog

$$
\begin{aligned}
\tilde{\mathscr{H}}(z)= & \sup _{v \in U} H\left(\bar{z}, x^{*}(z), v, p(z), \lambda_{0}\right) \geqq H\left(z, x^{*}(z), v_{2}, p(z), \lambda_{0}\right) \\
= & H\left(z, x^{*}(z), v_{2}, p(z+0), \lambda_{0}\right) \\
& -\sum_{i \in I_{\left(z, x^{*}\right)}}^{\Sigma_{i}} \mu_{i}(\{z\})^{\circ} \varphi\left(z, x^{*}(z), v_{2}\right) g_{i \xi}\left(z, x^{*}(z)\right) .
\end{aligned}
$$


Einerseits ist wegen $v_{2} \in \mathscr{H}^{-v}(z+0)$ nun $H\left(z, x^{*}(z), v_{2}, p(z+0), \lambda_{0}\right)=\tilde{\mathscr{H}}(z+0)$, andererseits gilt wegen $v_{2} \in W_{2}(z)$

$$
\begin{aligned}
& \mu_{i}(\{z\}) \varphi\left(z, x^{*}(z), v_{2}\right) g_{i \xi}\left(z, x^{*}(z)\right) \leqq-\mu_{i}(\{z\}) g_{i \ell}\left(z, x^{*}(z)\right) \\
& i \in I\left(z, x^{*}\right) \text { und damit } \\
& \sum_{\in \in\left(z, x^{*}\right)} \mu_{i}(\{z\}) \varphi\left(z, x^{*}(\dot{z}), v_{2}\right) g_{i \xi}\left(z, x^{*}(z)\right) \leqq \sum_{i \in I\left(z, x^{\circ}\right)}-\mu_{i}(\{z\}) g_{i t}\left(z, x^{*}(z)\right) .
\end{aligned}
$$

Deshalb habèn wir

$$
\tilde{\mathscr{H}}(z) \geqq \tilde{\mathscr{H}}(z+0)+\sum_{i \in I\left(z, x^{*}\right)} \mu_{i}(\{z\}) g_{i t}\left(z ; x^{*}(z)\right)
$$

Daraus folgt wegen (10) die erste Behauptung des, Satzes, d. h.

$$
\tilde{\mathscr{H}}(z)=\tilde{\mathscr{H}}(z+0)+\sum_{i \in I\left(z, x^{*}\right)} \mu_{i}(\{z\}) g_{i l}\left(z, x^{*}(z)\right) \text {. }
$$

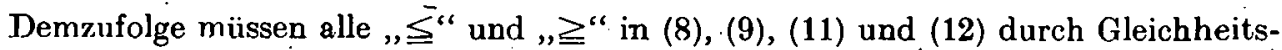
zeichen ersetzt, werden. Aus (8)- entsteht dadurch $H\left(z, x^{*}(z), v_{1}, p(z+0), \hat{\lambda}_{0}\right)$ $=\tilde{\mathscr{H}}(z+0)$, d. h. $v_{1} \in \mathscr{H}^{-v}(\dot{z}+0)$ für alle $v_{1} \in M_{1}(z)$. Also gilt $M_{1}(z) \subset \mathscr{H}^{-0}(z+0)$. Analog kommt $M_{2}(z) \subset \mathscr{H}^{-D}(z)$ durch das Gleichheitszeichen in (11) zustande. Die übrigen Behauptungen in a) ergeben sich aus (9) und (12).

c) $v_{1}$ sei beliebig aus $M_{1}(z)(\neq \emptyset)$. Ist $v_{1} \in \partial U$, so folgt aus (6) und a) $\mu_{i}(\{z\})=0$ für alle $i \in I\left(z, x^{*}\right)$. Damit ist $p(z)=p(z+0)$, d. h. $p$ ist in $z$ stetig. Ist $v_{1} \in$ int $U$, so folgt einerseits wegen $v_{1} \in \cdot M_{1}(z) \subset \mathscr{H}^{-v}(z)$ '(nach der Definition) und $M_{1}(z)$ $\subset \mathscr{H}^{-v}(z+0)($ nach $\left.a)\right)$.

$$
H_{v_{s}}\left(z, x^{*}(z), v_{1}, p(z), \lambda_{0}\right)=H_{v}\left(z, x^{*}(z), v_{1}, p(z+0), \lambda_{0}\right)=0
$$

für alle $j=1,2, \ldots, r$. Andererseits ist

Insgesamt erhalten wir

$$
\begin{aligned}
H_{v},\left(z, x^{*}(z), v_{1}, p(z), \lambda_{0}\right)= & H_{v},\left(z, x^{*}(z), v_{1}, p(z+0), \lambda_{0}\right) \\
& -\sum_{i \in I\left(z, x^{*}\right)} \mu_{i}(\{z\}) \varphi_{v_{j}}\left(z, x^{*}(z), v_{1}\right) g_{i \varepsilon}\left(z, x^{*}(z)\right) .
\end{aligned}
$$

$$
\sum_{i \in I\left(z, x^{*}\right)} \mu_{i}(\{z\}) \varphi_{v_{1}}\left(z, x^{*}(z), v_{1}\right) g_{i \xi}\left(z, x^{*}(z)\right)=0 \text {. }
$$

Wegen (7) muß $\mu_{i}(\{z\})=0$ für alle $i \in I\left(z, x^{*}\right)$ sein, d. h. $p$ ist in $z$ stetig

Bemerkung: Für die lineare Aufgábe

$$
\begin{aligned}
& \int_{t_{0}}^{t_{1}}\left(f_{0}(t, x(t))+f_{1}(t, x(t)) u(t)\right) d t \rightarrow \inf !, \\
& \dot{x}(t)=C(t) x(t)+D(t) u(t) ; \\
& |u(t)| \leqq 1, \quad,|x(t)| \leqq \alpha \quad \text { für } \quad t \in\left[t_{0}, t_{1}\right], \quad-x\left(t_{0}\right)=x\left(t_{1}\right)=0
\end{aligned}
$$

und die quasireguläre Aufgabe

$$
\begin{aligned}
& \int_{l_{0}}^{t_{1}}\left(f_{0}(t, x(t))+f_{1}(t, x(t)) u(t)+f_{2}(t, x(t)) u^{2}(t)\right) d t \rightarrow \text { inf } !, \\
& \dot{x}(t)=C(t) x(t)+D(t) u(t), \\
& |u(t)| \leqq 1, \quad|x(t)| \leqq \alpha \text { für } t \in\left[t_{0}, t_{1}\right], \quad x\left(t_{0}\right)=x\left(t_{1}\right)=0
\end{aligned}
$$


lauten die Bedingungen (6) und (7)

$$
C(t)( \pm \alpha)+D(t) \neq 0, \quad C(t)( \pm \alpha)-D(t) \neq 0, \quad D(t) \neq 0
$$

Diese Bedingungen können in vielen Fällen erfüllt werden. Dagegen gilt die Bedingung der àllgemeinen Lage von W. G. BolTJaNSKI nicht, wie am Anfang schon gesagt wurde. In [4] und [6] wurde gezeigt, daß bei einem optimalen Prozeß der Aufgabe (13) eine Aufsprungstelle im allgemeinen nicht zugleich eine Absprungstelle ist. Das heißt man kann die Theorie von H. W. KNoвLoch nicht für (13) anwenden. Für (14) ist die Forderung von H. W. KNoвLосн ebenfalls nicht erfüllt, denn nach. [6] bzw. Lemma 8 gilt $\dot{x}^{*}(z)=0$ für alle Aufsprung- bzw. Absprungstellen $z$.

Im allgemeinen besagt die Bedingung (6): Eine optimale Trajektorie kann mit Steuerungen aus dem Rande des Steuerbereichs $U$ nicht weiterhin auf dem Rande des Zustandsbereichs, $G$ bleiben.

\section{Einige Anwendungen}

In diesem Abschnitt werden aus den vorangegangenen Ergebnissen einige Schlußfolgerungen über die Optimalstellenmenge der Pontrjaginfunktion und über die Stetigkeit der optimalen Steuerung gezogen.

Lemma 8: Gegeben sei $\mathscr{H}^{-v}(z)=\{\bar{v}\}$ (d. h. $\mathscr{H}^{-v}$ besitzt genau ein Element) und $I\left(z, x^{*}\right) \neq \emptyset$. Dann gilt:

a) $\left(1, \varphi\left(z, x^{*}(z), v\right)\right) \operatorname{grad} g_{i}\left(z, x^{*}(z)\right)=0$ für alle $i \in I\left(z, x^{*}\right)$,

b) Unter (6) ist $\bar{v} \in$ int $U$ und $H_{\imath}\left(z, x^{*}(z), \vec{v}, p(z), \lambda_{0}\right)=0$.

Beweis: a) Nach Lemma 7 und Definition gilt

$$
\emptyset \neq M_{1}(z)=W_{1}(z) \cap \mathscr{H}^{-0}(z)=W_{1}(z) \cap\{\bar{v}\} \subset\{\bar{v}\} .
$$

Daraus folgt $\bar{v} \in W_{1}(z)$, d. h. $\varphi\left(z, x^{*}(z), \bar{v}\right) g_{i \epsilon}\left(z, x^{*}(z)\right) \geqq-g_{i t}\left(z, x^{*}(z)\right)$ für alle $i \in I\left(z ; x^{*}\right)$. Nach Lemma 7 und Satz 2 gilt $\emptyset \neq M_{2}(z) \subset \mathscr{H}^{-v}(z)=\{\bar{v}\}$. Deshalb ist $\{\bar{v}\}=M_{2}(z)=\dot{W}_{2}(z) \cap \mathscr{H}^{-v}(z+0)$. Daraus folgt $\bar{v} \in W_{2}(z)$, d. h. $\varphi\left(z, x^{*}(z), \bar{v}\right)$ $\times g_{i \xi}\left(z, x^{*}(z)\right) \leqq-g_{i t}\left(z, x^{*}(z)\right)$ für alle $i \in I\left(z, x^{*}\right)$. Also gilt $\left(1, \varphi\left(z, x^{*}(z), v\right)\right)$ $\times \operatorname{grad} g_{i}\left(z, x^{*}(z)\right)=0$ für alle $i \in I\left(z, x^{*}\right)$.

b) Aus (6) folgt sofort $\bar{v} \in$ int $U$ und wegen $v \in \mathscr{H}^{-v}(z)$ auch $H_{v}\left(z, x^{*}(z), \bar{v}, p(z), \lambda_{0}\right)$ $=0$ 【

Die Voraussetzung des Lemmas 8 ist bei den quasirégulären Aufgaben, beispielsweise (14), erfïllt. Weiterhin kann man Lemma 8 zur Untersuchung der folgenden quasilinearen Aufgabe der optimalen Steuerung anwenden:

$$
\begin{aligned}
& \int_{t_{0}}^{t_{1}}\left(f_{0}(t, x(t))+\sum_{j=1}^{r} f_{j}(t, x(t)) u_{j}(t)\right) d t \rightarrow \inf !, \\
& \dot{x}(t)=\varphi_{0}(t, x(t))+\sum_{j=1}^{r} \varphi_{j}(t, x(t)) u_{j}(t) \in \mathbf{R}^{n}, \\
& \beta_{j} \leqq u_{j}(t) \leqq b_{j}, \quad \alpha_{i} \leqq x_{i}(t) \leqq a_{i}, \quad x_{i}\left(t_{0}\right)=x_{i 0}, \quad x_{i}\left(t_{1}\right)=x_{i 1} \\
& \left(j=1,2, \ldots, r ; i=1,2, \ldots, n ; t \in\left[t_{0}, t_{1}\right]\right) .
\end{aligned}
$$


Lemma 9: Sei $\left(x^{*}, u^{*}\right)$ ein optimuler Prozeß der Aufgabe (16) und. (6) sei erfüllt. Dann existiert für beliebiges $z \in\left[t_{0}, t_{1}\right]$ mit $I\left(z, x^{*}\right) \div 0$ ein $j(1 \leqq j \leqq r)$ mit. $H_{v_{j}}\left(z, x^{*}(z), u^{*}(z), p(z), \lambda_{0}\right)=0$.

Beweis: Wäre die Behauptung falsch, dann gälte

$$
I_{v},\left(z, x^{*}(z), u^{*}(z), p(z), \lambda_{0}\right) \neq 0
$$

für $j=1,2, \ldots, r$. Für diese Aufgabe ist $H_{v}$ unabhängig von $v$, deshalb ist

$$
H_{v_{s}}\left(z, x^{*}(z), v, p(z), \lambda_{0}\right) \neq 0 \text {. }
$$

fuir alle $v \in U$ und $j=\overline{1}, 2, \ldots, r$. Daraus folgt wegen der Definition von $U$ und der Lincarität, von $H$ in $v$, daß $\mathscr{H}^{-v}(z)$-genau ein Element $\bar{v}$ enthält, d. h. $H\left(z, x^{*}(z), \cdot, \dot{p}(z), \lambda_{0}^{\circ}\right)$ hat genau eine Maximalstelle. Nun wird Lenima 8 angewandt, und es ergibt sich daraus $H_{v,}\left(z, x^{*}(z), \bar{v}, p(z), \lambda_{0}\right)=0$ für alle $j=1,2, \ldots, r$ im Widerspruch zu (17). Also muß die Behauptung des Lemmas rịchtig sein

I.emma 10: Für die Aufgabe (1) seien (4)-(7) erfüllt und es sei vorausgesetzt, duß $H\left(t, x^{*}(t), \cdot, p(t), \lambda_{0}\right)$ für alle $t \in\left[i_{0}, t_{1}\right]$ genau eine Maximalstelle besitzt. Dann ist die optimale Steuerung $u^{*}$ stetig.

Beweis: Nach Satz 2 ist $p$ stetig. Daraus folgt die Stetigkeit von $\widetilde{H}(\cdot, v)$ für alle $v \in U$ und damit wegen $\tilde{\mathscr{H}}(t)=\sup \tilde{H}(t, v)$ auch die Stetigkeit von $\tilde{\mathscr{H}}$ (vgl. B. N. Pšsenıčný [8: Kap. 3]). Wegen

$$
\mathscr{H}^{-v}(l)=\{v \in \dot{U} \mid \tilde{H}(t, v)=\tilde{\mathscr{H}}(t)\}
$$

ist $\mathscr{H}^{-v}$ nach Lemma 1 oberhalbstetig. Weil $\mathscr{H}^{-v}$ nach Voraussetzung einelementig ist, etwa $\mathscr{H}^{-v}(t)=\{\bar{u}(t)\}$, folgt aus der Oberhalbstetigkeit von $\mathscr{H}^{-v}$ die Stetigkeit ron $\bar{u}$. Nach $(3)$ gilt $u^{*}(t) \in \mathscr{H}^{-v}(t)$ fast überall in $\left[t_{0}, t_{1}\right]$, also stimmt $u^{*}$ mit $\bar{u}$ fast überall überein: Aus der Stetigkeit von $\bar{u}$, der rechtsseitigen Stetigkeit in $\left[t_{0}, t_{1}\right]$ und der Stetigkeit in $t_{1}$ von $u^{*}$ folgt, da $\beta u^{*}$ und $\bar{u}$ überall in $\left[t_{0}, t_{1}\right]$ übereinstimmen und damit $u^{*}$ stetig ist

Insbesondere erfïllt die Aufgabe (14) - (15) alle Voraussetzungen des Lemmas 10, deshalb ist jede optimale Steuerung dieser Aufgäbe stetig.

\section{LITERATUR}

[1] Boltuanski, W. G.: Mathematische Methoden der optimalen Steuerung. Akad. Verlagsges. Geest \& Portig K.-G.: Leipzig 1971.

[2] Ioffe, A. D., und V. M. Trchommov: Theorie der Extremala ufgaben (Ubers. a. d. Russ.). Dt. Verlag Wiss.: Berlin 1979:

[3] K мовцосн, H. W.: Das Pontryaginsche Maximumprinzip für Probleme mit Zustandsbeschränkung. ZAMM 55 (1975), $545-556$ und $621-634$.

[4] Hò̀va XUẦ PHÚ: Lineare Steuerungsprobleme mit engen Zustandsbereichen. Math. Operationsforsch. Statist., Ser. Optimization (eingereicht).

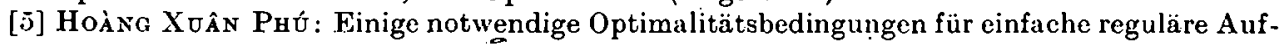
gaben der optimalen Steuerung. Z. Anal. Anw. (erscheint).

[6] Hòsa XUÂN PHú: Methoden zur Lösung von Aufgaben der optimalen Steuerung mit engen Zustandsbereichen. Dissertation A: Karl-Marx-Universität Leipzig 1983. 
[7] Hodxg X̃ûx Phú : Lösung einer einfachen rfgulären Aufgabe der optimalen Steuerung mit engen Zustandsbereichen anhand der Methode der Bereichsanalyse. Math. Operationsforsch. Statist., Ser. Optimization (eingereicht).

[8] Pšeničsyu, B. N.: Notwendige Optimalitätsbedingungen. BSB B. G. Teubner Verlagsgesellschaft: Leipzig 1972.

[9] Rademacher, H.: Uber partielle und totale Differenzierbarkeit van Funktionen mehrerer Variablen und über Transformation der Doppelintegrale. Math. Ann. 79 (1919), 340-359.

Hanuskripteingang: 15.07 .1983

\section{VERFASSER :}

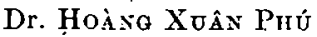

Sektion Mathematik der Karl-Marx-Universität

DDR - 7010 Leipzig, Karl-Marx-Platz

Heimatadresse: Truong Dang cao cap Nguyen Ai Quoc

Tu liem

Ha noi, SR Victnam 